\title{
Structural and Electronic Properties of Nano Hydroxyapatite
}

\author{
A. ÖZTÜrK KIRAZ ${ }^{a, *}$, S. KAYA ${ }^{a}$ AND C. GÖK ${ }^{b}$ \\ ${ }^{a}$ Department of Physics, Faculty of Science and Arts, Pamukkale University, 20070, Denizli, Turkey \\ ${ }^{b}$ Department of Materials Science and Engineering, Faculty of Technology, \\ Pamukkale University, 20070, Denizli, Turkey \\ (Received March 7, 2019; in final form March 5, 2020)
}

\begin{abstract}
Nowadays, the uses of nanobiomaterials are increasing and one of the most concerning biomaterial is the hydroxyapatite (HAp). In this study, the electronic properties of the nano hydroxyapatite were studied theoretically using density functional theory. Calculations were carried out on Gaussian 09 package program using the B3LYP functional method, 6-31G(d,p) basis set. We determined the fitting geometry, highest occupied molecular and lowest unoccupied molecular orbital energy, molecular electrostatic potential, electrostatic surface potential, and electronic properties of the nano hydroxyapatite from the calculations. The spectroscopic values of the nano-HAp were also obtained using ab initio computational method. Infrared and Raman spectroscopic data acquired via this method were compared with the literature results. These computational studies on nano hydroxyapatite provide a framework for materials design and selection for biomaterials used in many areas.
\end{abstract}

DOI: 10.12693/APhysPolA.137.1017

PACS/topics: nano hydroxyapatite, DFT, FTIR, MEP

\section{Introduction}

Calcium hydroxyapatite $\left[\mathrm{HAp}, \mathrm{Ca}_{10}\left(\mathrm{PO}_{4}\right)_{6}(\mathrm{OH})_{2}\right]$ is the initial inorganic component of the teeth enamel [1]. Hydroxyapatite (HAp) is the naturally occurring calcium phosphate and member of apatite mineral family. HAp has recently attracted attention because it has been used as a biomaterial for unnatural bone since it has perfect features in the context of osteocompatibility and high absorbability $[1,2]$. HAp has been studied and used as an environmentally benign functional material with adsorbent materials through changing cations or adsorbing immutable ones on the crystal surface. HAp can be created from both artificial and genuine homelands [3]. Furthermore, nano hydroxyapatite (nano-HAp) has wider unique surface area, better mechanical properties and advance osteoconductivity compared to conventional hydroxyapatite [4].

In consequence of the fundamental function fulfilled by HAp, great significance has been supposed from its lattice dynamics to get information about its structure at atomic level. Various experimental methods, like X-ray diffraction [5, 6], IR and Raman spectroscopy [7-9] and nuclear magnetic resonance (NMR) [10] have been performed for unadulterated substance and after submerge in simulated body fluid (SBF) to clarify HAp molecule. However, different computational studies using molecular mechanics $[11,12]$ and quantum mechanical methods $[13,14]$ have been carried out because of the problems related to explaining the experimental results, dynamical properties of the lattice and electronic properties.

Recently, density functional theory (DFT) methods are widely used to determine the large sized molecules at

*corresponding author; e-mail: aslio@pau.edu.tr low computational cost [15-24]. In the literature, experimental and theoretical studies on the characteristic of the HAp are rare. Quantum mechanical calculations using DFT methods are generally utilized to investigate the characterization of the materials and compounds [25, 26].

In this paper, we declared the synthesis, properties and molecular structure of the nano-HAp. Structural, spectroscopic and electronic parameters of the HAp were determined through the DFT methods. In addition, the Fourier transform infrared spectroscopy (FTIR) and Raman spectroscopy were studied to compare with theoretical calculations. Consequently, debates on the empirical and computational studies of this structure spearhead a better knowledge of the biomaterial of HAp.

\section{Materials and methods}

All the chemicals and reagents used for the experiments and the analysis in this study were of analytical grade and used without further purification. Calcium nitrate tetrahydrate $\mathrm{Ca}\left(\mathrm{NO}_{3}\right)_{2} \cdot 4 \mathrm{H}_{2} \mathrm{O}$ and diammonium hydrogen phosphate $\left(\mathrm{NH}_{4}\right)_{2} \mathrm{HPO}_{4}$ was used for the preparation of hydroxyapatite. An amount of $25 \mathrm{ml}$ of $10 \mathrm{mmol}\left(\mathrm{NH}_{4}\right)_{2} \mathrm{HPO}_{4}$ solution was dropwise added to hydroxyapatite mixture. The mixture was aged for $3 \mathrm{~h}$ at room temperature, after mechanical stirring for $1 \mathrm{~h}$ at $50^{\circ} \mathrm{C}$. $\mathrm{pH}$ of the solution during stirring was maintained above 10 . Then, the precipitate was separated by filtration. The resulting precipitate was rinsed with distilled water until the wash water was neutral. Finally, nanohydroxyapatite materials were dried at $105^{\circ} \mathrm{C}$ for $3 \mathrm{~h}$.

FTIR spectrum was recorded by a Perkin-Elmer spectrum one model FTIR spectrophotometer $\left(3700-400 \mathrm{~cm}^{-1}\right)$ and the Raman spectrum was recorded by BTC665N-785S-SYS model Raman spectrometer for the characterization of HAp. 
Density functional theory was used to perform the quantum mechanical calculations of the HAp molecule in gas phase. All the calculations were carried out via the Gaussian 09.C1 [27] package program using the B3LYP functional method, $6-31 \mathrm{~g}(\mathrm{~d}, \mathrm{p})$ basis set $[28,29]$ and GaussView 5.0.9 [30] was used for the visualization of the structure, simulated vibrational spectra, and electronic properties.

\section{Results}

\subsection{Molecular properties}

The chemical formula of the nano-HAp is shown in Fig. 1a. The optimized geometry at B3LYP/6-31G(d,p) level and the atom numbering scheme of the nano-HAp is presented in Fig. 1b, respectively.

\subsection{Structural properties}

The optimization results like bond distance, bond angle and dihedral angle of the nano-Hap were determined from B3LYP level by using 6-31G(d,p) basis set for all atoms. The molecular formula of the nano-HAp has 44 bond distances, 19 bond angles and 10 dihedral angles. These bond distances and bond angles are presented in Table I. Our theoretical results such as bond distances and bond angles correspond with the empirical and calculated values given in the literature. As presented in Table I, determined bond distances and bond angles help us know the molecular structure of the nano-HAp. The length of the P1-O2 is reported to be 1.541 $\AA$ [31] while it is computed as $1.54 \AA$ at B3LYP level by using $6-31 \mathrm{G}(\mathrm{d}, \mathrm{p})$ basis set. O2-P1-O3 bond angle is calculated as 104.02 by using DFT methods. For all molecule specific and similar structural parameters, determined bond distances and bond angles featuring the nano-HAp molecule were calculated at B3LYP/6-31G(d,p) level.

Today, for the identification of molecular structures, spectroscopic procedures are generally utilized to investigate the structures of the molecule, to follow the reactions and to control the pureness of the compounds. The necessary methods for the chemical characterization are the infrared and the Raman spectroscopy. One of the

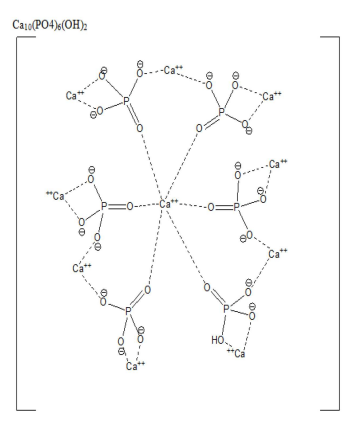

(a)

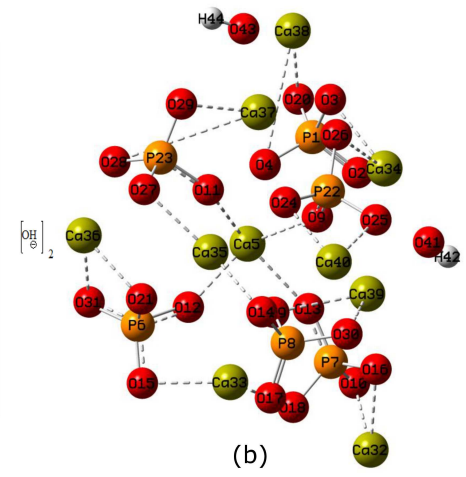

(b)
Fig. 1. The crystal structure of the nano-HAp.
Bond distances and bond angles of nano-HAp.

TABLE I

\begin{tabular}{l|c|c}
\hline \hline \multicolumn{1}{c|}{ Bond } & Distances $[\AA]$ & Lit. values $[\AA]$ \\
\hline P1-O2 & 1.54 & $1.54[31]$ \\
P1-O3 & 1.66 & $1.63[31]$ \\
P1-O4 & 1.57 & $1.54[31]$ \\
P1-O20 & 1.56 & $1.54[31]$ \\
Ca5-O9 & 2.50 & $2.31-2.41[32]$ \\
Ca5-O11 & 2.37 & $2.31-2.41[32]$ \\
Ca5-O12 & 2.33 & $2.31-2.41[32]$ \\
Ca5-O13 & 2.41 & $2.31-2.41[32]$ \\
O14-Ca35 & 2.55 & $2.31-2.41[32]$ \\
O15-Ca33 & 2.32 & $2.31-2.41[32]$ \\
O41-H42 & 0.96 & $0.97[31]$ \\
O43-H44 & 0.96 & $0.97[31]$ \\
\hline Bond & Angles [ $\left.{ }^{\circ}\right]$ & Lit. values [ $\left.{ }^{\circ}\right]$ \\
\hline O2-P1-O3 & 104.02 & $135.28[33]$ \\
O3-P1-O4 & 102.05 & $135.28[33]$ \\
O4-P1-O20 & 113.26 & $135.28[33]$ \\
O3-P1-O20 & 103.21 & $135.28[33]$ \\
O9-Ca5-O11 & 75.06 & $84-132[32]$ \\
O9-Ca5-O12 & 125.72 & $84-132[32]$ \\
O9-Ca5-O13 & 80.82 & $84-132[32]$ \\
O11-Ca5-O12 & 90.72 & $84-132[32]$ \\
O14-Ca35-O27 & 143.55 & $84-132[32]$ \\
O15-Ca33-O18 & 134.75 & $84-132[32]$ \\
O39-O41-H42 & 117.69 & $104.29[34]$ \\
O37-O43-H44 & 104.53 & $104.29[34]$ \\
\end{tabular}

most practical instruments for the chemical compounds in terms of both theoretical calculations and experimental working is the analysis of vibrational spectroscopy. In recent years, the vibrational frequencies calculated via ab initio computational methods are becoming significant instruments. In order to get the spectroscopic values of the nano-HAp, we executed frequency calculations. The analysis of the calculations of vibrational wave numbers and groups of the molecules are illustrated in Table II. It enables us to assign the fundamental vibrations. The empirical results are also given in the same table to compare them with the calculated corresponding results. The literature data given in Tables II and III are consistent with the values obtained in our experimental studies.

The significant intense band arises from the phosphate $\nu 1$ symmetric stretching $(\mathrm{P}-\mathrm{O}$ stretching $)$ at $\approx 996 \mathrm{~cm}^{-1}$ in the Raman spectrum of the structure. O-O stretching at $1007 \mathrm{~cm}^{-1}$ and $\mathrm{Ca}$ effects are around $500 \mathrm{~cm}^{-1}$. The theoretical and experimental Raman activities of the nano-HAp can clearly be seen in Table III.

\subsection{Electronic properties}

In order to analyze the chemical bonding in more details, we performed density functional theory electronic structure calculations for nano-HAp. Highest occupied molecular orbital (HOMO) is defined as the ability to give an electron while lowest unoccupied molecular orbital (LUMO) is described as the ability to obtain 
Theoretical and experimental values of vibration energies.

TABLE II

\begin{tabular}{|c|c|c|c|}
\hline \multicolumn{2}{|c|}{ Wave number $\left[\mathrm{cm}^{-1}\right]$} & \multirow{2}{*}{ Groups } & \multirow{2}{*}{ Experimental } \\
\hline Exp. & Theor. & & \\
\hline 3570 & 2900 & $-\mathrm{O}-\mathrm{H}^{-}$groups & Refs. [35-37] \\
\hline 3432 & 2600 & stretching vibrations of structural $-\mathrm{O}-\mathrm{H}$ & Refs. [35-37] \\
\hline $3200-3600$ & 2500 & free hydrogen bands and free $-\mathrm{O}-\mathrm{H}$ groups & Refs. [38-40] \\
\hline $2800-2900$ & $1800-2700$ & $-\mathrm{P}-\mathrm{O}$ groups bending vibrations & Refs. [38-41] \\
\hline 1642 & & vibrational mode of planes bending of $\mathrm{O}-\mathrm{H}$ & Ref. [40] \\
\hline 1384 & 1500 & $\mathrm{PO}_{4}^{3-}$ groups & Refs. [38-40] \\
\hline $1033-1095$ & $900-1200$ & stretching vibrations of phosphate groups $\left(\mathrm{PO}_{4}^{3-}, \mathrm{P}-\mathrm{O}\right)$ & Refs. $[35,38-41]$ \\
\hline $600-400$ & $290-614$ & bending vibrations and translational mode of phosphate groups $\left(\mathrm{PO}_{4}^{3-}, \mathrm{O}-\mathrm{P}-\mathrm{O}\right)$ & Refs. $[38,40,42,43]$ \\
\hline
\end{tabular}

Theoretical and experimental Raman shifts.

TABLE III

\begin{tabular}{c|c|l|l}
\hline \hline \multicolumn{2}{c|}{ Raman shift $\left[\mathrm{cm}^{-1}\right]$} & \multicolumn{1}{|c}{ Groups } & \multirow{2}{*}{ Experimental } \\
\hline Theor. & Exp. & & \multicolumn{1}{|c}{ Refs. [44-46] } \\
\hline $2434.96-2506.23$ & $2500-3000$ & $-\mathrm{O}-\mathrm{H}^{-}$groups & Refs. [40, 44, 45, 47] \\
$911.28-894.96$ & 960 & bending vibration of $-\mathrm{P}-\mathrm{O}$ groups & Refs. [35, 46-48] \\
$953.23-934.66$ & & $\begin{array}{l}\mathrm{PO}_{4}^{-3} \text { groups } \\
\text { bending vibrations of }\left(\mathrm{PO}_{4}^{3-}, \mathrm{O}-\mathrm{P}-\mathrm{O}\right)\end{array}$ & Refs. [40, 44, 45]
\end{tabular}

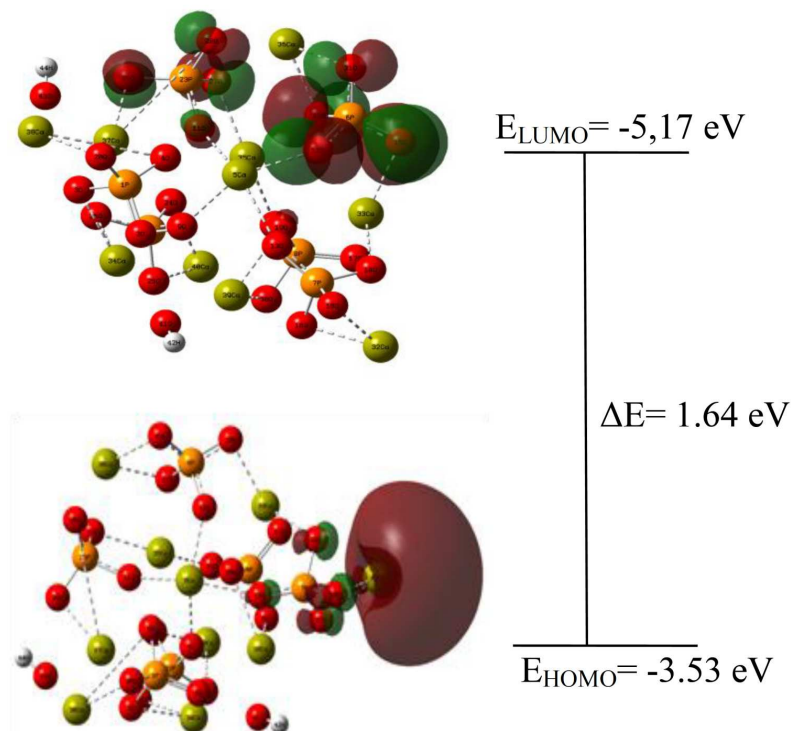

Fig. 2. The atomic orbital compositions of the HAp.

an electron [49]. The gap between HOMO-LUMO energies plays an important role in the molecule chemical stability and also represents the chemical hardness-softness of the molecule, electronegativity and chemical activity. A molecule with small HOMO-LUMO energy gap is called a soft molecule, and it is more polarizable and has high chemical reactivity and low kinetic stability [50].

A larger HOMO-LUMO gap of an electronic system should be less active than a smaller HOMO-LUMO gap [51] also called hard molecule. The HOMO and LUMO energies are determined as -5.17 and $-3.53 \mathrm{eV}$ at the B3LYP/6-31G(d,p) level. As seen from Fig. 2 and electronic parameters represented in Table IV, the LUMOs are located around the $\mathrm{Ca}$ atom while the HOMOs are located around the $\mathrm{P}$ atoms. The energy gap value between HOMO and LUMO of the HAp molecule is $1.64 \mathrm{eV}$. These small values are the proof of the high probability of the charge transfer in the molecule [52].

The band gap of HAp was measured as $3.95 \mathrm{eV}$ by photoluminescence measurement. Several individual energy states were also found in the range (2.6-3.9) eV [53]. In some of these articles, the band gap of HAP was calculated between 4.51 and $5.4 \mathrm{eV}[54,55]$. Generally, it is well known that a DFT calculation underestimates the value of the energy gap compared with the energy band gap. Therefore, it is so difficult to comment on the experimental and theoretical energy gap of the HAp structure [56].

\subsection{Molecular surfaces}

Molecular electrostatic potential (MEP) and electrostatic surface potential (ESP) were used to foretell the active sides of the molecule and the hydrogen bonding interplay as well as their potential use in biological identification studies [57, 58]. MEP and ESP were modeled at the B3LYP $/ 6-31 \mathrm{G}(\mathrm{d}, \mathrm{p})$ level to predict the active sites and the hydrogen bonding interplays. The potential changes from -2.617 a.u. to +2.617 a.u. of the HAp molecule has two possible sides for nucleophilic attack, as can easily be seen in Fig. 3. The molecule has positive region while most of the molecule has negative regions. The negative regions are mainly over the entire molecule especially in Ca cations. These negative red colored regions represent the molecule which can interact with another molecule or a group over the $\mathrm{PO}_{4}$. 
TABLE IV

The electronic parameters of the nano-HAp.

\begin{tabular}{l|c}
\hline \hline Electronic parameters & B3LYP $/ 6-31 \mathrm{G}(\mathrm{d}, \mathrm{p})$ \\
\hline$E_{\mathrm{HOMO}}[\mathrm{eV}]$ & -5.17 \\
$E_{\mathrm{LUMO}}[\mathrm{eV}]$ & -3.53 \\
$\Delta E=E_{\mathrm{LUMO}}-E_{\text {HOMO }}[\mathrm{eV}]$ & 1.64 \\
$I[\mathrm{eV}]$ & 5.17 \\
$A[\mathrm{eV}]$ & 3.53 \\
$\chi[\mathrm{eV}]$ & -4.35 \\
$\eta[\mathrm{eV}]$ & 0.82 \\
$S[1 / \mathrm{eV}]$ & 0.60 \\
$\mu[\mathrm{eV}]$ & 4.35 \\
$\omega[\mathrm{eV}]$ & 11.53
\end{tabular}
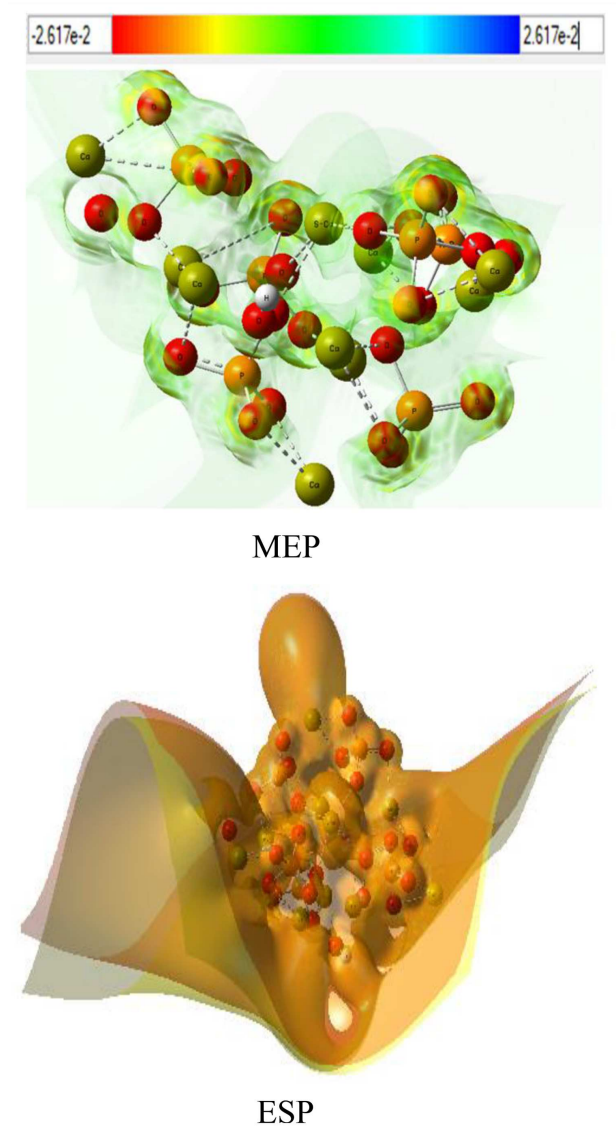

Fig. 3. Molecular electrostatic potential (MEP) and electrostatic surface potential (ESP) surface of HAp obtained from B3LYP/6-31G(d,p).

\section{Conclusion}

In this paper, a classical interatomic force field for nano-HAp has been obtained from density functional theory calculations based on a B3LYP and 6-31G $(\mathrm{d}, \mathrm{p})$ basis set quality. Firstly, structural parameters of the nanoHAp such as bond lengths and bond angles have been calculated with a good agreement of the experimental results in the literature. Secondly, it is very important to know the electronic properties for the determination of apatite binding properties. It is known from the literature that if the energy gap is high between the HOMO and LUMO orbital, chemical stability of molecule will be highly strong. In this study, the energy difference between HOMO and LUMO orbitals of the HAp molecule is $1.64 \mathrm{eV}$. This small value points out the proof of the high probability of the charge transfer in the molecule. With this result, the nano-HAp can be called soft molecule apparently. Thirdly, we also have set our results against the IR and Raman with the convenient similar empiric FT-IR magnetic nano-HAp values given in the literature and our measured Raman spectrum data of the nanoHAp. These results have a good agreement with the other literature values as shown in Tables II and III, respectively. Finally, the results of ESP calculation indicate that total charge of this molecule is neutral and total electron density has located over the molecule. Negative region has been around the $\mathrm{Ca}$ atoms, and positive region has been over the $\mathrm{PO}_{4}$ and free $\mathrm{O}-\mathrm{H}$ groups. Because of that, the molecule will be active over the $\mathrm{O}-\mathrm{H}$ and $\mathrm{O}-\mathrm{P}$. If the biomaterials are desired to be doped in the molecule, this part of molecule will be active for the interaction. With the importance of the nano-HAp, the scope of such a derivation is to apply it in further computationally demanding studies related to the comprehension of the geometric and electronic features of HAp. As a result, this study can be also applied to a variety of biomolecules related hydroxyapatite and can enhance our understanding of the nature and behavior of biomaterials used in many areas.

\section{Acknowledgments}

The authors are grateful to PAUBAP (Project No. 2012BSP004 and 2018FEBE002). Some of the computations were performed using TUBITAK/ULAKBIM clusters and Theoretical Physics clusters of Pamukkale University. Some of the experimental measures (Raman spectrum) were performed in the Chemical and Physical Laboratory of Pamukkale University and IR spectrum measured in the Chemical Laboratory of Ege University.

\section{References}

[1] R.A. Young, W.E. Brown, Biological Mineralization and Demineralization, Springer-Verlag, New York 1982.

[2] H. Yan, H. Wang, S. Gong, J. Radioanal. Nucl. Chem. 292, 545 (2011).

[3] C. Gok, J. Radioanal. Nucl. Chem. 301, 641 (2014).

[4] R. Hou, G. Zhang, G. Du, D. Zhan, Y. Cong, Y. Cheng, J. Fu, Coll. Surf. B Biointerfaces 103, 318 (2013).

[5] K. Il-Seok, P.N. Kumta, Mater. Sci. Eng. B 111, 232 (2004). 
[6] R. Murugan, S. Ramakrishna, J. Cryst. Growth $\mathbf{2 7 4}$ 209 (2005).

[7] I. Rehman, W. Bonfield, J. Mater. Sci. Mater. Med. 8, 1 (1997).

[8] H. Tsuda, J. Arends, J. Dent. Res. 73, 1703 (1994).

[9] R. Cusco, F. Guitia, S. de Aza, L. Artus, J. Eur. Ceram. Soc. 18, 1301 (1998).

[10] X. Qiu, Z. Hong, J. Hu, L. Chen, X. Chen, X. Jing, Biomacromolecules 6, 1193 (2005).

[11] W.T. Lee, M.T. Dove, E.K.H. Salje, J. Phys. Condens. Matter 12, 9829 (2000).

[12] N.H. de Leeuw, Phys. Chem. Chem. Phys. 6, 1860 (2004).

[13] L. Calderin, M.J. Stott, A. Rubio, Phys. Rev. B 67, 134101 (2003).

[14] N.H. de Leeuw, Phys. Chem. Chem. Phys. 4, 3865 (2002).

[15] M. Karabacak, E. Sahin, M. Cinar, I. Erol, M. Kurt, J. Mol. Struct. 886, 148 (2008).

[16] X. Xuan, J. Wang, Y. Zhao, J. Zhu, J. Raman Spectrosc. 38, 865 (2007).

[17] M. Karabacak, A. Coruh, M. Kurt, J. Mol. Struct. 892, 125 (2008).

[18] N. Sundaraganesen, C. Meganathan, B.D. Joshua, P. Mani, A. Jayaprakash, Spectrochim. Acta A 71, 1134 (2008)

[19] N. Sundaraganesen, C. Meganathan, B.D. Joshua, Spectrochim. Acta Part A 69, 871 (2008).

[20] X. Xuan, C. Zhai, Spectrochim. Acta A 79, 1663 (2011).

[21] M.R. Anoop, P.S. Binil, S. Suma, M.R. Sudarsnakumar, S. Mary, Y.H.T. Vaghese, C. Yohannan Pnicker, J. Mol. Struct. 969, 48 (2010).

[22] A. Atac, M. Karabacak, C. Karaca, E. Köse, Spectrochim. Acta A 85, 145 (2012).

[23] M. Karabacak, Z. Cinar, M. Kurt, S. Sudha, N. Sundaraganesan, Spectrochim. Acta A 85, 179 (2012).

[24] P.B. Nagabalasubramanian, M. Karabacak, S. Periandy, Spectrochim. Acta A 85, 43 (2012).

[25] M. Kurt, T.R. Sertbakan, M. Ozduran, M. Karabacak, J. Mol. Struct. 921, 178 (2009).

[26] H. Chermette, Coord. Chem. Rev. 178-180, 699 (1998).

[27] C. Corminboeuf, F. Tran, J. Weber, J. Mol. Struct. Theochem. 762, 1 (2006).

[28] M.J. Frisch, G.W. Trucks, H.B. Schlegel et al., Gaussian 09 (now Gaussian 16), Gaussian Inc., Wallingford (CT) 2016.

[29] R. Dennington, T. Keith, J. Millam Gauss View, Version 5.0.9, Semichem Inc., 2009.

[30] C. Adamo, V. Barone, J. Chem. Phys. 110, 6158 (1999).

[31] K.P. Huber, G. Herzberg, Constants of Diatomic Molecules in: Molecular Spectra and Molecular Structure, Van Nostrand Reinhold Co., 1979.
[32] M.D. Dadmun, W.A. Van Hook, D.V. Noid, Y.B. Melnichenko, B.G. Sumpter, Computational Studies, Nanotechnology and Solution Thermodynamics of Polymer Systems, Kluwer Academic, New York 2000, p. 132.

[33] K. Kawaguchi, S. Saito, E. Hirota, J. Chem. Phys. 82, 4893 (1985).

[34] A. Charo, F. De Lucia, J. Mol. Spectrosc. 94, 426 (1982).

[35] J.P. Barone, Ph.D. Thesis, State University of New York, Buffalo 1976.

[36] F. Freund, R.M. Knobel, J. Chem. Soc. Dalton Trans. 11, 1136 (1977).

[37] L. Wienand, M.J. Dallemagne, G. Duyckaerts, Nature 190, 164 (1961).

[38] B.O. Fowler, Inorg. Chem. 13, 194 (1974).

[39] W.E. Klee, G. Engel, J. Inorg. Nucl. Chem. 32, 1837 (1970).

[40] S. Koutsopoulos, J. Biomed. Mater. Res. 62, 600 (2002).

[41] S.J. Gadaleta, R. Mendelsohn, E.P. Paschalis, N.P. Camacho, F. Betts, A.L. Boskey, in: Mineral Scale Formation and Inhibition, Ed. Z. Amjad, Plenum Press, New York 1995, p. 283.

[42] J. Arends, M.R. Christoffersen, H. Eckert, B.O. Fowler, J.C. Heughebaert, G.H. Nancollas, J.P. Yesinowski, S.J. Zawacki, J. Cryst. Growth 84, 515 (1987).

[43] J.J. Stutmann, J.D. Termine, A.S. Posner, Trans. NY Acad. Sci. 27, 669 (1965).

[44] G.R. Sauer, W.B. Zunic, J.R. Durig, R.E. Wuthier, Calcif. Tissue Int. 54, 414 (1994).

[45] H. Tsuda, J. Arends, J. Dent. Res. 72, 1609 (1993).

[46] W.P. Griffith, J. Chem. Soc. A, 286 (1970).

[47] D.C. O'Shea, M.L. Bartlett, R.A. Young, Arch. Oral Biol. 19, 995 (1974).

[48] P.N. de Aza, F. Guitian, C. Santos, S. de Aza R. Cusco, L. Artus, Chem. Mater. 9, 916 (1997).

[49] İ. Kara, Y. Kara, A. Öztürk Kiraz, R. Mammadov, Spectrochim. Acta A 149, 592 (2015).

[50] K. Fukui, Science 218, 747 (1982).

[51] H. Pir Gümüş, Ö. Tamer, D. Avcı, Y. Atalay, Spectrochim. Acta A 129, 219 (2014).

[52] R. Kurtaran, S. Odabasoglu, A. Azizoglu, H. Kara, O. Atakol, Polyhedron 26, 5069 (2007).

[53] G. Rosenman, D. Aronov, L. Oster, J. Haddad, G. Mezinskis, I. Pavlovska, M. Chaikina, A. Karlo, J. Lumin. 122-123, 936 (2007).

[54] P. Rulis, L. Ouyang, W.Y. Ching, Phys. Rev. B 70 , 155104 (2004).

[55] K. Matsunaga, A. Kuwabara, Phys. Rev. B 75, 014102 (2007).

[56] M. Tsukada, M. Wakamura, N. Yoshida, T. Watanabe, J. Mol. Catal. A Chem. 338, 18 (2011).

[57] J.S. Murray, K. Sen, Concepts and Applications, Elsevier, Amsterdam 1996.

[58] E. Scrocco, J. Tomasi, Adv. Quantum Chem. 11, 115 (1978). 